\title{
Interactive comment on "Photooxidation of pinonaldehyde at ambient conditions investigated in the atmospheric simulation chamber SAPHIR" by Michael Rolletter et al.
}

\section{Anonymous Referee \#2}

Received and published: 12 May 2020

The paper reports experiments on pinonaldehyde oxidation, conducted in the SAPHIR chamber in Juelich. In one experiment, the oxidation was initiated exclusively by photolysis, in the other, pinonaldehyde reacted primarily with $\mathrm{OH}$. The chamber is highly instrumented and has been very well characterized. The experiments were conducted at low $\mathrm{NO}$ and concentrations of pinonaldehyde, $\mathrm{HCHO}$, acetone, $\mathrm{NO}, \mathrm{NO} 2, \mathrm{O} 3, \mathrm{HONO}$, $\mathrm{OH}$ and $\mathrm{HO} 2$ were measured, together with the actinic flux outside the chamber, over a period of $\sim 4 \mathrm{~h}$. The results were compared with simulations based on the master chemical mechanism (MCM) and on a theoretically based alternative. The results show that there are significant deficiencies in both these mechanisms and suggestions for revisions are made. 
The results and the analysis make a significant contribution to improving our understanding of an aspect of the atmospheric oxidation of $\alpha$-pinene - the key pinonaldehyde precursor - and should be published. The authors should consider the following points in a revision of the manuscript.

It is disappointing that only two experiments were conducted, one on photolysis and the other on $\mathrm{OH}$ oxidation, so there is no testing of the observations and interpretation via variation, for example, of the initial [NO]. I appreciate that the experiments are complex and resource-intensive, but some comment might be made on the implications of this limitation. In addition, the authors should comment on the strong variations in [HO2] in the photolysis experiment(Figs 2 and 6) which is greater than the experimental uncertainty and is not reproduced in the models. The afternoon decrease is at least partly explained by a decrease in $\mathrm{j}$ (pinonaldehyde), but there are other variations also. The variability is much less pronounced in the $\mathrm{OH}$ initiation experiments.

It would be helpful if there were an indication of just how important pinonaldehyde is in the oxidation of $\alpha$-pinene. There are a number of routes to pinonaldehyde and their importance depends on conditions, so this is not an easy request to satisfy, but some indication under relevant conditions would be useful, together with an indication of the dominant routes. It is suggested that the yield is small in the field campaigns discussed on $\mathrm{p} 16$. Under what conditions is pinonaldehyde production important? Some information on the transmission of the chamber walls and its impact on the spectral distribution should be made. The maximum in the pinonaldehyde spectrum lies below $300 \mathrm{~nm}$, while the absorption has fallen to half its maximum value at $\sim 310 \mathrm{~nm}$, so the photolysis wavelengths and rate could be impacted significantly by the wall transmission and its wavelength dependence.

The photolysis experiment demonstrates that the MCM significantly underestimates the photolysis rate and this is corrected in the paper, which also demonstrates that the yields of acetone and $\mathrm{HCHO}$ are substantially overestimated in the MCM. This is addressed by using the mechanism developed by Fantechi et al to describe the

Printer-friendly version

Discussion paper 
reactions of the oxy radical formed from pinonaldehyde photolysis. As pointed out in the paper, their simulations did not include the subsequent reactions of the main products of this reaction, 4-Hydroxynorpinonaldehyde, which will react further and will also be photolyzed, almost certainly on the timescales of the experiment. It is suggested that it might be a further source of $\mathrm{HO} 2$, but it could also be a source of $\mathrm{HCHO}$ and acetone. Further discussion, possibly ruling out this possibility, and some suggestion of the likely products is essential. The estimated experimental yield of acetone shown in Fig 4 is initially negative, which is difficult to understand. Some explanation is needed.

The MCM and the Fantechi mechanism cannot explain the yields of $\mathrm{HCHO}$ or acetone in the $\mathrm{OH}$ initiated oxidation and the paper shows that they seriously underestimate HO2. Two attempts are made to understand the deficiencies, based on isomerization of RO2 species via an autoxidation mechanism and photolysis of intermediates. The latter appears to provide a better explanation. It is disappointing that a more considered analysis isn't given, especially since the senior author of the Fantechi paper leads the theoretical kinetics group at Juelich. I am not suggesting that major calculations are appropriate at this stage, but more informed comments and suggestions of the way forward might be made (e.g. amplifying the comment at lines 8,9 on p 14).

Smaller comments:

What integrator was used in the simulations?

The English needs some attention (e.g. line 12, p12 should be rise (or increase would be better); line 5, p13 replace "MCM like found in" with "MCM, such as the")

Line 15, p15. Should be version 3.3.1.

Interactive comment on Atmos. Chem. Phys. Discuss., https://doi.org/10.5194/acp-2020-310, 2020. 